\title{
Multi-dimensional digital human models for ergonomic analysis based on natural data representations
}

\author{
Niels C.C.M. Moes \\ Department of Industrial Design Engineering, \\ Delft University of Technology, \\ Delft, Netherlands \\ E-mail: ccm.moes@xs4all.nl
}

\begin{abstract}
Digital human models are often used for ergonomic analysis of product designs, before physical prototypes are available. However, existing digital human models cannot be used to simultaneously: 1) consider the tissue loads and the physiological effects of the tissue loads; 2) optimise the product properties. This paper develops multi-dimensional digital human models for ergonomic analysis based on natural data representations, which include anatomy, morphology, behaviour, physiology, tissue, and posture data representations. The results show that the multi-dimensional digital human models can be used to: 1) accelerate the design process; 2) assess mechanical and physiological loads inside the body and in the contact area between the body and the product; 3 ) optimise the quality of the product; 4) reduce the number of user trials needed to create the product.
\end{abstract}

Keywords: human modelling; ergonomics; product design; design process.

Reference to this paper should be made as follows: Moes, N.C.C.M. (2015) 'Multi-dimensional digital human models for ergonomic analysis based on natural data representations', Int. J. Digital Human, Vol. 1, No. 1, pp.72-80.

Biographical notes: Niels C.C.M. Moes is an Associate Professor at the Faculty of Industrial Design Engineering, Delft University of Technology, The Netherlands. He received his MSc at the Eindhoven University of Technology in 1974. He earned his PhD from the Delft University of Technology in 2004. His primary research interests include the human factors aspects in human modelling and in applying ubiquitous technologies in product design and design education. Since Spring 2014, he has retired.

This paper is a revised and expanded version of a paper entitled 'Digital human body modelling to support designing products for physical interaction' presented at International Design Conference - Design 2006, Dubrovnik, Croatia, 15-18 May 2006. 


\section{Introduction}

Digital human models are often used for ergonomic analysis of product designs, before physical prototypes are available. The digital human models should allow designers to:

1 accelerate the design process

2 assess mechanical and physiological loads inside the body and in the contact area between the body and the product

3 optimise the quality of the product

4 reduce the number of user trials needed to create the product.

However, existing digital human models cannot be used to simultaneously:

1 consider the tissue loads and the physiological effects of the tissue loads

2 optimise the product properties.

Existing digital human models for medical analysis of patient conditions can be used to consider the tissue loads and the physiological effects of the tissue loads. Existing digital human models for ergonomic analysis of product designs can be used to optimise the product properties. Therefore, this paper develops multi-dimensional digital human models for ergonomic analysis, which combine elements of existing digital human models for medical analysis of patients with elements of existing digital human models for ergonomic analysis of product designs.

The multi-dimensional digital human models are based on natural data representations, which include anatomy, morphology, behaviour, physiology, tissue, and posture data representations. Therefore, the multi-dimensional digital human models are more knowledge intensive than existing digital human models. As a result, the multi-dimensional digital human models can be used to simultaneously:

1 consider the tissue loads and the physiological effects of the tissue loads

2 optimise the product properties.

The goal of this paper is to develop the multi-dimensional digital human models in a step-wise manner, and to use the multi-dimensional digital human models to:

1 analyse the internal stresses and deformations, tissue relocations, and muscle activations and the resulting effects on the physiological tissue functions under external loads

2 use the analysis results to optimise the product.

The knowledge that is needed in specific multi-dimensional digital human models depends on the applications at hand. Therefore, the goal of this paper is to determine:

1 what knowledge is needed to build adaptive quasi-organic models of the human body

2 how to manage this knowledge.

As a result, this paper presents:

1 the requirements for the multi-dimensional digital human models 
2 the knowledge that is needed to build the multi-dimensional digital human models

3 the procedures that are needed to build the multi-dimensional digital human models,

4 the initial implementation results.

\section{The requirements for the multi-dimensional digital human models}

The multi-dimensional digital human models must be capable of representing different individual humans, or different groups of individual humans. The multi-dimensional digital human models are based on algorithms that process and relate the knowledge. The knowledge is typically incomplete for individual humans, and the knowledge typically varies for individual humans.

Therefore, the multi-dimensional human models must be adaptive quasi-organic models of the human body, which consider variable properties such as the shape and size of the body, the shape and size of the internal tissues, the material properties of the internal tissues, and the physiological functioning of the internal tissues.

Consequently, the multi-dimensional digital human models must consist of frameworks and sub-models, which can be added to or removed from the frameworks, and which can be adapted for different individual humans, or different groups of individual humans. The multi-dimensional digital human models can only consider the knowledge which is available. Therefore, the multi-dimensional digital human models must also be extendable. The frameworks and sub-models must be capable of adding new knowledge, when new knowledge is available.

\section{The knowledge that is needed to build the multi-dimensional digital human models}

Figure 1 shows the structure of the multi-dimensional digital human models. The multi-dimensional digital human models consist of frameworks and sub-models, which can be added to or removed from the frameworks, and which can be adapted for different individual humans, or different groups of individual humans.

The frameworks do not contain the knowledge. The sub-models deliver the knowledge. Therefore, the frameworks use algorithms to:

1 process the knowledge that is delivered by the sub-models

2 facilitate communication between the sub-models

3 make specific decisions.

The sub-models deliver different types of knowledge: anatomy, morphology, behaviour, physiology, tissue, and posture knowledge. Anatomy knowledge consists of the internal structures, the active and passive elements, the physical locations, the physical functions, and the functional relationships. Morphology knowledge consists of the shapes, the connections, and the contact properties (the geometric relationships). Physiology knowledge consists of the functions of the fluids (the blood, lymph, and interstitial fluids), the soft tissues (the muscle, and adipose tissues), the hard tissues (the bone tissues), the metabolic processes, and the nerve systems. The behaviour knowledge 
consists of the material properties (the elastic, nonlinear, and viscous properties), and the muscular structures. The posture knowledge consists of the joint positions, the changes in the joint positions, the changes in the shapes of the body, the changes in the shapes of the tissues, the relocations of the tissues, and the changes in the forces in the body.

Figure 1 The structure of the multi-dimensional digital human models

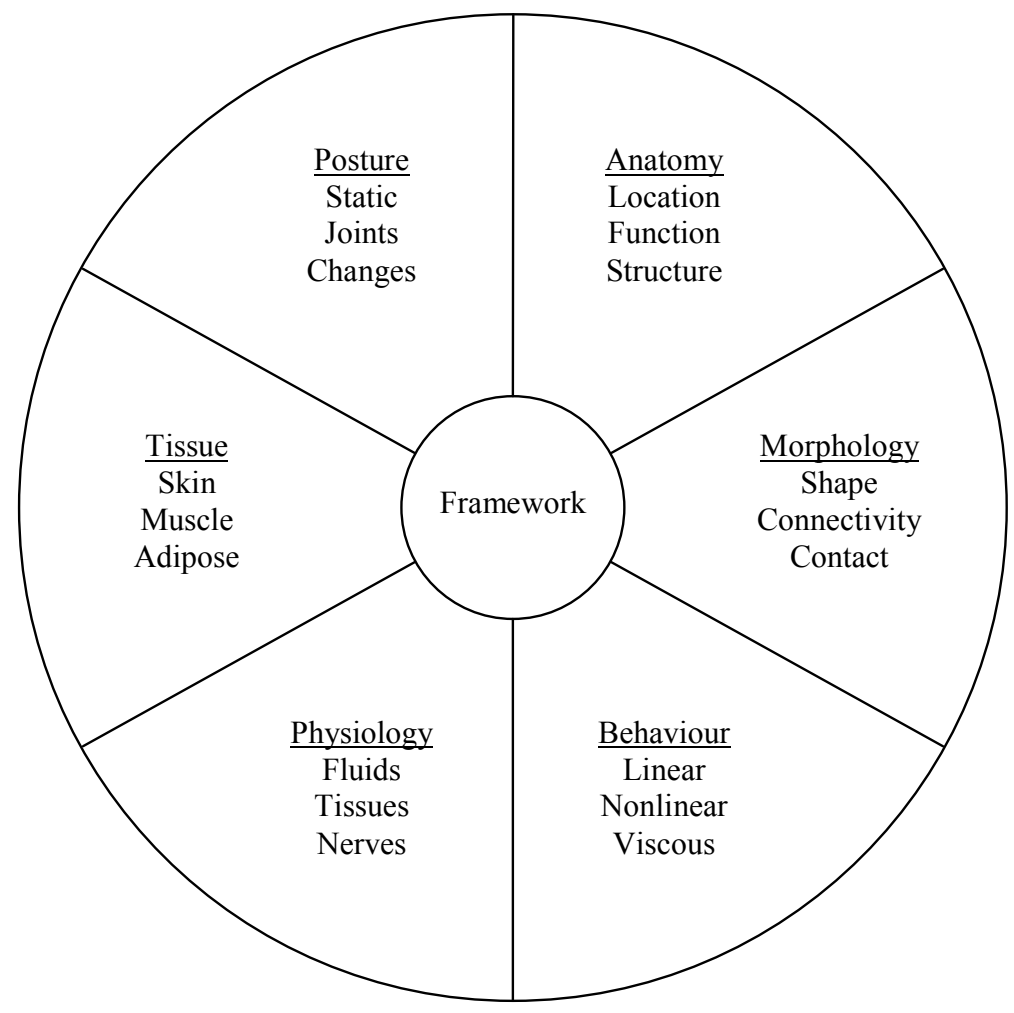

New sub-models, which are not shown, can be added, when new sub-models are available. New knowledge, which is not shown, can also be added, when new knowledge is available.

\subsection{The procedures that are needed to build the multi-dimensional digital human models}

Figure 2 shows the procedures that are needed to build the multi-dimensional digital human models. The procedures consist of measurement, reduction, formalisation, instantiation, and utilisation procedures. The procedures can also be grouped into measurement procedures (which are used to capture the knowledge about the humans), conceptualisation procedures (which are used to transform the knowledge into the algorithms) and implementation procedures (which are used to transform the algorithms into the digital human models). The procedures are used to sequentially transform the knowledge into a more useable format. The end result is multi-dimensional digital human 
models that can be used to analyse the interactions between the multi-dimensional digital human models and accordingly modelled products.

Figure 2 The procedures that are needed to build the multi-dimensional digital human models

\begin{tabular}{|c|c|c|}
\hline $\begin{array}{l}\text { Measurement } \\
\text { procedures }\end{array}$ & $\begin{array}{l}\text { Conceptualisation } \\
\text { procedures }\end{array}$ & $\begin{array}{l}\text { Implementation } \\
\text { procedures }\end{array}$ \\
\hline & $\begin{array}{c}\text { Reduction } \\
\text { procedures } \\
\text { Formalisation } \\
\text { procedures }\end{array}$ & $\begin{array}{l}\text { Instantiation } \\
\text { procedures } \\
\text { Utilisation } \\
\text { procedures }\end{array}$ \\
\hline
\end{tabular}

\subsubsection{The measurement procedures}

The measurement procedures consist of physical or conceptual procedures which are used to capture measured knowledge (which is not very useable). For example, the measurement procedures consist of physical (laser scanning) or conceptual (database access) procedures which are used to capture measured knowledge (scanned point clouds) that describes the shapes of individual humans, or groups of individual human.

\subsubsection{The reduction procedures}

The reduction procedures consist of statistical or conceptual procedures which are used to transform the measured knowledge (which is not very usable) into the structured knowledge (which is more usable). For example, the reduction procedures consist of statistical or conceptual (vague discrete interval modelling) procedures (Moes et al., 2001; Rusák, 2003) which are used to transform the measured shape knowledge (scanned point clouds) for individual humans, or groups of individual humans, into structured shape knowledge [limited sets of characteristic surface points), for individual humans, or groups of individual humans (Moes, 2004)].

\subsubsection{The formalisation procedures}

The formalisation procedures consist of statistical or mathematical procedures which are used to transform the structured knowledge (which is not very understandable) into relationship knowledge (which is more understandable). For example, the formalisation procedures consist of statistical or mathematical (anatomical, physiological, biomechanical) procedures which are used to transform the structured shape knowledge (limited sets of characteristic surface points) into relationship shape knowledge (relationships between the external environmental conditions, the external forces, and the locations of the characteristic surface points).

The formalisation procedures also consist of conceptual procedures which are used to transform the relationship knowledge (which is not very executable) into algorithmic knowledge (which is more executable). For example, the formalisation procedures also consist of conceptual (algorithm development) procedures which are 
used to transform the relationship shape knowledge (relationships between the external environmental conditions, the external forces, and the locations of the characteristic surface points) into algorithmic shape knowledge (algorithms which can be converted into software and executed on digital computers, within the morphology sub-models of the multi-dimensional digital human models). The resulting algorithmic shape knowledge can be used to rotate, translate, and align the scanned point clouds, by matrix operations, with limited sets of characteristic points, analyse the resulting rotated, translated, and aligned point clouds to create inner and outer hulls, and convert the resulting inner and outer hulls into shape models of distribution trajectories and statistically defined location indices (Moes, 2004). Therefore, the resulting algorithmic shape knowledge can be used to:

1 describe the shapes of individual humans, or groups of individual humans

2 generate new shapes, based on the external environmental conditions, the external forces, and the locations of the characteristic surface points).

\subsubsection{The implementation procedures}

The implementation procedures consist of conceptual procedures which are used to transform the algorithmic knowledge (which is not very executable) into implemented knowledge (which is more executable). For example, the implementation procedures consist of conceptual (software development) procedures which are used to transform the algorithmic shape knowledge (algorithms which can be converted into software and executed on digital computers, within the morphology sub-models of the multi-dimensional digital human models) into implemented knowledge (implemented morphology sub-models of the multi-dimensional digital human models), In order to support the computation the mathematical expressions are converted to algorithms, and suitable software is used for the actual implementation.

The implementation procedures also consist of test procedures which are used to find and fix errors in measured, structured, relationship, algorithmic, and implemented knowledge. For example, the implementation procedures also consist of test procedures which are used to find and fix errors in measured, structured, relationship, algorithmic, and implemented knowledge for the morphology sub-models of the multi-dimensional digital human models.

\subsubsection{The utilisation procedures}

The utilisation procedures consist of conceptual procedures which use the resulting multi-dimensional digital human models to optimise product properties based on ergonomics criteria. For example, the utilisation procedures consist of conceptual (software simulation) procedures which use the multi-dimensional digital human models to optimise the shapes of chairs, by changing product properties (design parameters) to reduce physical stresses, based on an objective optimisation function (OOF), such as the ergonomics goodness index (EGI) (Moes and Horváth, 2002a). As a result, the utilisation procedures consist of conceptual (software simulation) procedures which use the multi-dimensional digital human models to optimise product properties, and to improve specific user-product interactions. 


\section{The initial implementation results}

The procedures described in this paper were used to create a multi-dimensional digital human model for the lower torso and upper leg regions of the human body. The measurement procedures used physical (laser scanning) and conceptual [visible human project database access (VHP, 1997)] procedures to capture measured knowledge (scanned point clouds) which described the shapes of the skin and bones of individual humans, or groups of individual humans, when sitting on chairs. The reduction procedures used vague discrete interval modelling (VDIM) procedures to transform the measured shape knowledge (scanned point clouds) for individual humans, or groups of individual humans, into structured shape knowledge (limited sets of characteristic surface points), for individual humans, or groups of individual humans, when sitting on chairs. The formalisation and implementation procedures were used to transform the structured shape knowledge (limited sets of characteristic surface points) for individual humans, or groups of individual humans, when sitting on chairs, into generic morphology and behaviour sub-models for the multi-dimensional digital human model. New geometric alignment (Moes, 2004) software, new VDIM (Rusák, 2003) software, and commercially available statistical analysis software were used to create the sub-models for the multi-dimensional digital human model.

The multi-dimensional digital human model was used to predict the shapes of body surfaces and bones for individual humans, or groups of individual humans, when sitting in chairs, in terms of distributed spatial points (Moes, 2004). The morphology model was used to predict the shapes of the tissues and the connectivities between the tissues, based on the contact conditions. The behaviour model was used to predict the effects of the external environmental conditions, and the external forces, on the predicted shapes. The predicted shapes were used to create solid finite element models (FEMs). The solid FEMS were used to validate the constitutive equations by comparing computed pressure distribution knowledge (Moes and Horváth, 2002b) with measured pressure distribution knowledge (Moes, 2006) for individual humans, or groups of individuals, when sitting in chairs. Therefore, the multi-dimensional digital human model was used to analyse the relationships between the stresses and strains inside the bodies of individual humans, or groups of individual humans, and the shapes of chairs, based on actual measured knowledge, and the results were used to create virtual models of the optimised chairs (Moes, 2004). Commercially available statistical analysis software and commercially available finite element analysis (FEM) software (MARC, 2001) were used to test the multi-dimensional digital human model. The constitutive models for the mechanical behaviour of human tissues are quite complex. Therefore, the commercially available statistical analysis software and the commercially available finite element analysis (FEM) software was used to test many different constitutive equations (Moes, 2004).

Figure 3 shows one FEM and three chair models. The three chair models differ only in shape, and one chair is a flat surface. The three chairs were modelled as rigid bodies. The three chair models were used to create loads for the finite element (FEM) model, which was used to predict internal stresses, strains, and tissue relocations for an individual human, when sitting in the three chairs. 
Figure 3 One FEM and three chair models

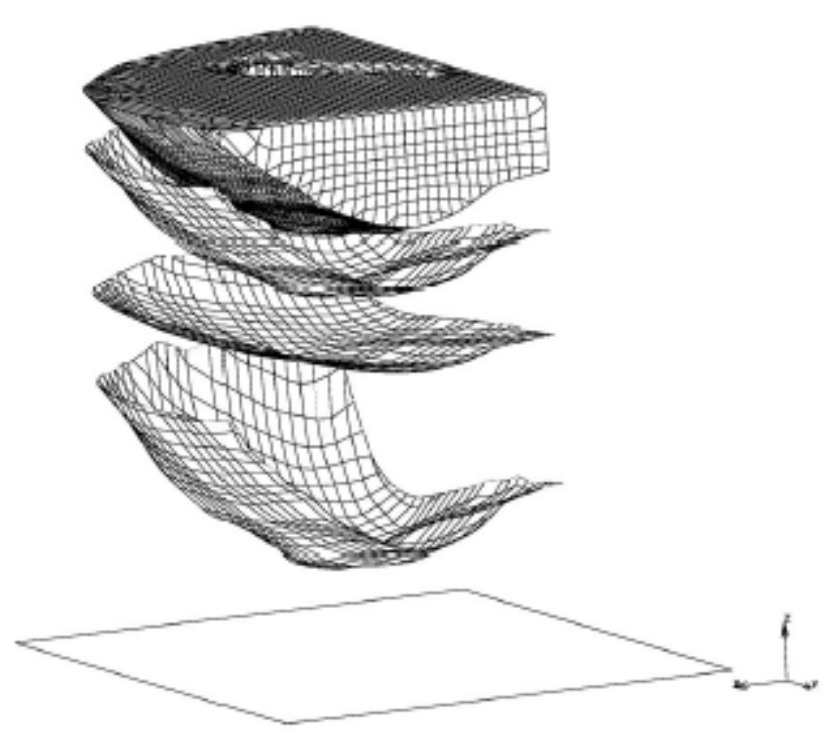

\section{The results and conclusions}

The results (Moes, 2004) show that the procedures described in this paper can be used to create multi-dimensional digital human models. The results show that the multi-dimensional digital human models described in this paper can be used to predict shape knowledge for individual humans, or groups of individual humans, when sitting in chairs. Therefore, the results show that the multi-dimensional digital human models described in this paper can be used to optimise the shapes of chairs, by changing product properties (design parameters) to reduce physical stresses, based on an OOF. As a result, the results show that the procedures and the multi-dimensional digital human models described in this paper are feasible, and the results describe significant technical contributions for one specific application (sitting in chairs).

However, more work is needed to create and test complete multi-dimensional digital human models for other specific applications. More work is needed to create statistical and mathematical relationships for complete multi-dimensional digital human models for other specific applications. More work is needed to create algorithms and software for complete multi-dimensional digital human models for other applications. Further research is needed to:

1 improve the framework

2 improve the sub-models and create new sub-models

3 testing and optimise the multi-dimensional digital human models

4 use the multi-dimensional digital human models for actual design tasks. 


\section{References}

MARC (2001) MARC Volume A: Theory and Users Guide, MARC Analysis Research Corporation, Palo Alto, CA.

Moes, C.C.M. (2004) Advanced Human Body Modelling to Support Designing Products for Physical Interaction, Delft University of Technology, ISBN: 90-018829-0 [online] http://repository.tudelft.nl/assets/uuid:75a23948-7bbd-4ce4-93de-defe41d10af7/dep_moes_ 20041213.pdf.

Moes, C.C.M. (2006) 'Modelling the sitting pressure distribution and the location of the points of maximum pressure for body characteristics and rotation of the pelvis', Ergonomics, submitted.

Moes, C.C.M. and Horváth, I. (2002a) 'Optimizing product shape with the Ergonomics Goodness Index, Part I: Conceptual solution', in McCabe Paul, T. (Ed.): Contemporary Ergonomics, pp.314-318, The Ergonomics Society, Taylor \& Francis.

Moes, C.C.M. and Horváth, I. (2002b) 'Estimation of the non-linear material properties for a finite elements model of the human body parts involved in sitting', in Lee, D.E. (Ed.).

Moes, C.C.M., Rusák, Z. and Horváth, I. (2001) 'Application of vague geometric representation for shape instance generation of the human body', in Mook, D.T. and Balachandran, B. (Eds.): Proceedings of DETC'01, Computers and Information in Engineering Conference, (CDROM: DETC2001/CIE-21298), ASME, Pittsburgh, Pennsylvania.

Rusák, Z. (2003) Vague Discrete Interval Modelling for Product Conceptualization in Collaborative Virtual Design Environments, Delft University of Technology, Fac. Industrial Design Engineering [online] http://repository.tudelft.nl/assets/uuid:7b6d2cc1-16cf-4337-a7affff25c880577/803627.pdf.

VHP (1997) The Visible Human Project [online] http://www.nlm.nih.gov/research/visible/ visible_human.html (accessed January 2006). 